\title{
Effectiveness of adalimumab in treating patients with active psoriatic arthritis and predictors of good clinical responses for arthritis, skin and nail lesions
}

\author{
F Van den Bosch, ${ }^{1}$ B Manger, ${ }^{2}$ P Goupille, ${ }^{3}$ N McHugh, ${ }^{4}$ E Rødevand, ${ }^{5}$ P Holck, ${ }^{6}$ R F van \\ Vollenhoven, ${ }^{7} \mathrm{M}$ Leirisalo-Repo, ${ }^{8} \mathrm{O}$ FitzGerald, ${ }^{9} \mathrm{M}$ Kron, ${ }^{10} \mathrm{M}$ Frank, ${ }^{10} \mathrm{~S}$ Kary, ${ }^{10}$ \\ H Kupper ${ }^{10}$
}

- Additional data

(supplementary tables 1 and 2 and supplementary appendix) are published online only at http://ard.bmj.com/content/ vol69/issue2

${ }^{1}$ University Hospital, Ghent, Belgium; ${ }^{2}$ Universität Erlangen/ Nürnberg, Erlangen, Germany; ${ }^{3}$ Université Francois Rabelais, Tours, France; ${ }^{4}$ Royal National Hospital, Bath, UK; ${ }^{5}$ St Olavs Hospital, Trondheim, Norway;

${ }^{6}$ Regionshospitalet Silkeborg,

Silkeborg, Denmark; ${ }^{7}$ The

Karolinska Institute, Stockholm

Sweden; ${ }^{8}$ University Central

Hospital, Helsinki, Finland; ${ }^{9} \mathrm{St}$ Vincent's University Hospital,

Dublin, Ireland; ${ }^{10}$ Abbott $\mathrm{GmbH}$ \& Co KG, Ludwigshafen, Germany

Correspondence to:

$\mathrm{F}$ van den Bosch, University Hospital Gent, Department of Rheumatology, De Pintelaan 185, B-9000 Gent, Belgium; filip. vandenbosch@ugent.be

Accepted 17 September 2009 Published Online First

7 October 2009

\section{ABSTRACT}

Objectives: To evaluate the effectiveness of adalimumab in patients with psoriatic arthritis (PSA) and identify predictors of good clinical response for joint and skin lesions.

Methods: Patients received adalimumab $40 \mathrm{mg}$ every other week in addition to standard therapy in this prospective, 12-week, open-label, uncontrolled study. Four definitions of good clinical response were used: $\geqslant 50 \%$ improvement in American College of Rheumatology response criteria (ACR50), good response according to European League Against Rheumatism (EULAR) guidelines, a $\geqslant 3$-grade improvement in Physician Global Assessment of psoriasis (PGA) and a $\geqslant 50 \%$ improvement in the Nail Psoriasis Severity Index (NAPSI). Response predictors were determined by logistic regression with backward elimination (selection level was $5 \%$ ). Results: Of 442 patients, $94 \%$ completed 12 weeks of treatment. At week $12,74 \%, 51 \%$ and $32 \%$ of the patients had achieved ACR20, 50 and 70, respectively; $87 \%$ and $61 \%$ experienced moderate and good responses according to EULAR criteria, respectively. The percentage of patients with PGA results of "clear/almost clear" increased from $34 \%$ (baseline) to $68 \%$. The mean NAPSI score was reduced by $44 \%$. No new safety signals were detected. A lower Health Assessment Questionnaire Disability Index (HAQ-DI) score, greater pain assessment, male sex and absence of systemic glucocorticoid therapy were strongly associated with achievement of ACR50 and good response according to EULAR criteria. In addition, greater C-reactive protein concentration and polyarthritis predicted ACR50, and non-involvement of large joints predicted a good response according to EULAR criteria. Conclusions: Adalimumab was effective in patients with PsA. Lower impairment of physical function, greater pain, male sex and no systemic treatment with glucocorticoids were factors that increased the chance of achieving a good clinical response.

Few studies have addressed whether predictors for a good clinical response to treatment with tumour necrosis factor (TNF) antagonists in patients with psoriatic arthritis (PsA) can be identified. The STEREO (for "SafeTy and Efficacy of adalimumab in patients with active psoriatic arthritis - an openlabel, multinational study to evaluate the Response to Every-Other week adalimumab when added to insufficient standard therapy including patients who failed prior treatment with other TNF inhibitors") trial prospectively evaluated the treatment effect of adalimumab in $>400$ patients with active PsA who were eligible for treatment with TNF antagonists in daily rheumatology practice. ${ }^{12}$ We also evaluated predictive factors for a good clinical response to adalimumab with respect to arthritis, skin and nail disease.

\section{METHODS}

\section{Patients}

Main inclusion criteria were: age $\geqslant 18$ years, PsA diagnosed by a rheumatologist, $\geqslant 3$ tender and $\geqslant 3$ swollen joints, previous treatment with $\geqslant 1$ disease-modifying antirheumatic drugs (DMARDs) and enrolment in accordance with each participating country's current guidelines for anti-TNF treatment of PsA. DMARDs, non-steroidal antiinflammatory drugs (NSAIDs), or oral glucocorticoids ( $\leqslant 10 \mathrm{mg}$ prednisolone equivalent/day), as well as topical psoriasis therapy, could be continued if the dosage was stable (additional details regarding the inclusion/exclusion criteria and study design are available in the supplementary material).

\section{Study design and measures}

STEREO was a prospective, open-label, uncontrolled study conducted in nine European countries. Patients self-administered adalimumab $40 \mathrm{mg}$ (Abbott Laboratories, Abbott Park, Illinois, USA) subcutaneously every other week for 12 weeks in addition to their pre-existing antirheumatic treatment. Patients who benefited from adalimumab therapy could continue up to week 20 if adalimumab was not commercially available. Observed data at week 12 were used for all effectiveness analyses. Presence or absence of dactylitis, defined as swelling of the entire finger or toe, and enthesitis at the heels were documented at baseline. Measures of effectiveness for PsA were at least $20 \%, 50 \%$ and $70 \%$ improvements in the American College of Rheumatology response criteria (ACR20, ACR50 and ACR70, respectively), ${ }^{3}$ tender joint count (TJC; 0-78 joints), swollen joint count (SJC; 0-76 joints), the 28-joint Disease Activity Score (DAS28) based on erythrocyte sedimentation rate (ESR; mm/first hour), ${ }^{4}$ moderate and good European League Against Rheumatism (EULAR) response criteria using the DAS28, ${ }^{5}$ and the PsA response criteria (PsARC), ${ }^{6}$ which was modified by using a 0-100 $\mathrm{mm}$ visual analogue scale (VAS) for the Physician or Patient Global Assessment of disease activity (PhGA or PaGA). Additional online under the BMJ Journals unlocked scheme, see http:// ard.bmj.com/info/unlocked.dtl 
measurements included 0-100 mm VAS for pain, the Health Assessment Questionnaire Disability Index (HAQ-DI; score of $0-3){ }^{7}$ and C-reactive protein (CRP) concentration ( $\mathrm{mg} / \mathrm{dl}$ ). Psoriasis was assessed by Physician Global Assessment (PGA) for psoriasis (a 7-point scale ranging from "clear" to "severe"), ${ }^{8}$ target lesion assessment (total plaque score 0-15, not assessed at week 2) requiring a lesion of $\geqslant 2 \mathrm{~cm}$ in the greatest diameter at baseline, ${ }^{8}$ Nail Psoriasis Severity Index (NAPSI; 0-80, only of the hands), ${ }^{9}$ and the Dermatology Life Quality Index (DLOI; score of 0-30). ${ }^{10}$ NAPSI and DLOI were evaluated only at baseline, week 12 and week 20 .

\section{Statistical analysis}

All patients who received at least one adalimumab injection were included in the analyses. Endpoints for good clinical responses at week 12 were achievement of ACR50, a good response according to EULAR criteria for PsA, ${ }^{11}{ }^{11} 12$ improvement by $\geqslant 3$ grades in PGA for psoriasis (evaluated in patients with PGA worse than "mild" at baseline) and a $\geqslant 50 \%$ improvement in NAPSI score for psoriatic nail disorder (evaluated in patients with NAPSI $\geqslant 10$ at baseline).

Continuous variables analysed as possible predictors of good clinical response for joint and skin/nail manifestations were age (per year), duration of PsA (per year), CRP (per mg/dl), PhGA and PaGA (per mm), pain (per mm); and DAS28 (per unit). In addition, HAQ-DI score (per unit) was evaluated for PsA, and duration of psoriasis (per year) and DLOI score (per unit) were evaluated for skin and nail disorders.

Categorical variables (yes vs no) analysed as possible predictors of good clinical response for joint and skin/nail manifestations were: male sex; dactylitis; enthesitis; prior TNF antagonist therapy; ongoing systemic treatment with $\geqslant 1$ DMARDs, with sulfasalazine (SSZ), or with glucocorticoids; history of tobacco use; $\geqslant 1$ inflamed large joint (knee, shoulder, elbow, hip); prior treatment with $>2$ DMARDs; polyarthritis ( $\geqslant 5$ swollen joints) versus oligoarthritis ( $<5$ swollen joints); and PGA > "moderate" versus PGA $\leqslant$ "moderate". For skin/nail manifestations only, ongoing topical treatment with glucocorticoids and prior ultraviolet A (UVA) and/or psoralen and UVA (PUVA) phototherapy were evaluated.

Crude odds ratios (ORs) with 95\% confidence intervals (CIs) and $p$ values based on two-sided $\chi^{2}$ tests (continuous variables) or Fisher exact tests (categorical variables) were calculated for all possible predictive factors. For the identification of predictors of ACR50 and good responses according to EULAR criteria, the ensemble of all factors was investigated by logistic regression with backward elimination (selection level was 5\%). The predictive value of the final model was assessed by calculating the area under the receiver operating characteristic (ROC) curve. For $\mathrm{a} \geqslant 3$-grade improvement in PGA and $\mathrm{a} \geqslant 50 \%$ improvement in NAPSI score, we separately investigated only the predictors because datasets for the complete ensemble of possible predictors were missing in $>20 \%$ of patients with skin and nail lesions; thus, a selection bias could not be excluded. All values presented are mean (SD) unless otherwise noted.

\section{RESULTS}

\section{Patient disposition, withdrawals and adalimumab treatment duration}

Of the 442 patients enrolled, 94\% completed week 12 and 39\% continued beyond week 12. During the complete treatment period, $6(1.4 \%)$ patients withdrew because of unsatisfactory therapeutic response and 26 (5.9\%) patients withdrew because of adverse events. Other reasons (withdrawal of consent, protocol violation, loss to follow-up, or other) are not shown. The mean adalimumab treatment duration was 103 days (median, 85 days).

\section{Patient characteristics at baseline}

Patient baseline characteristics are shown in table 1. All patients had psoriatic symptoms at baseline and/or a history of psoriasis. Plaque psoriasis (defined as PGA not "clear") was documented in 366 (82.8\%) patients, encompassing 206 patients with PGA greater than "mild" ("mild to moderate" in 59 patients, "moderate" in 82 patients, "moderate to severe" in 46 patients and "severe" in 19 patients). Psoriatic nail dystrophy (NAPSI > 0) was reported for 259 (59.3\%) patients, including 164 patients with a mean NAPSI of $\geqslant 10$.

\section{Effectiveness of adalimumab treatment}

ACR, EULAR and modified PsARC response rates at week 12, percentages of patients with PGA "clear" or "almost clear" and changes in target lesion and NAPSI score are shown in figs 1-4. The mean number of tender and swollen joints and mean DAS28, HAO-DI and DLOI scores were reduced by $61 \%, 73 \%$, $45 \%, 47 \%$ and $41 \%$, respectively, from baseline to week 12 . The supplementary material provides a detailed summary of effectiveness measures.

\section{Identification of predictors of a good clinical response} Predictors of good clinical response for PsA at week 12

The logistic regression with backward elimination revealed that a lower HAQ-DI score, male sex, no systemic therapy with glucocorticoids and a greater patient's assessment of pain were strongly associated with achievement of ACR50 and good response according to EULAR criteria (table 2). The area under the ROC curve was 0.72 for the final models of predictor identification for ACR50 and good response according to EULAR criteria. Results for all individual predictors analysed are shown in the supplementary material.

Predictors of good clinical response for psoriasis at week 12 Improvement of $\geqslant 3$ grades in PGA was experienced by $81(42 \%$, 13 missing) of the 206 patients with a baseline PGA greater than

Table 1 Baseline characteristics of patients with psoriatic arthritis (PsA) at baseline

\begin{tabular}{lc}
\hline & All patients (n= 442) \\
\hline Female, n (\%) & $221(50.0)$ \\
Median (SD) age, years & $47.8(11.5)$ \\
Mean (SD) PsA duration, years & $10.6(8.2)$ \\
Mean (SD) psoriasis duration, years & $19.4(12.9)$ \\
Rheumatoid factor positive, n (\%) & $51(11.6)$ \\
Anti-CCP antibody positive, n (\%) & $28(6.3)$ \\
HLA-B27 positive, n (\%) & $102(23.3)$ \\
Dactylitis, n (\%) & $131(29.7)$ \\
Enthesitis, n (\%) & $154(34.9)$ \\
Swollen joint count (0-66), median (quartile 1, quartile 3) & $8(5,13)$ \\
Mean (SD) DAS28 & $4.93(1.15)$ \\
Prior anti-TNF therapy, n (\%) & $66(14.9)$ \\
Ongoing DMARD therapy, $\mathrm{n}(\%)$ & $301(68.1)$ \\
Ongoing systemic glucocorticoid therapy*, n (\%) & $128(28.9)$ \\
\hline
\end{tabular}

*Maximum, prednisone equivalent $10 \mathrm{mg} /$ day.

CCP, cyclic citrullinated peptide; DAS28, 28-joint Disease Activity Score; DMARD, disease-modifying antirheumatic drug; HLA, human leukocyte antigen; TNF, tumour necrosis factor. 
Figure 1 Percentages of patients achieving American College of Rheumatology $20 \%, 50 \%$ and $70 \%$ response criteria (ACR20/50/70), moderate and good European League Against Rheumatism (EULAR) criteria responses and the modified psoriatic arthritis response criteria (mPsARC) at week $12(n=414)$. Data are from observed cases.

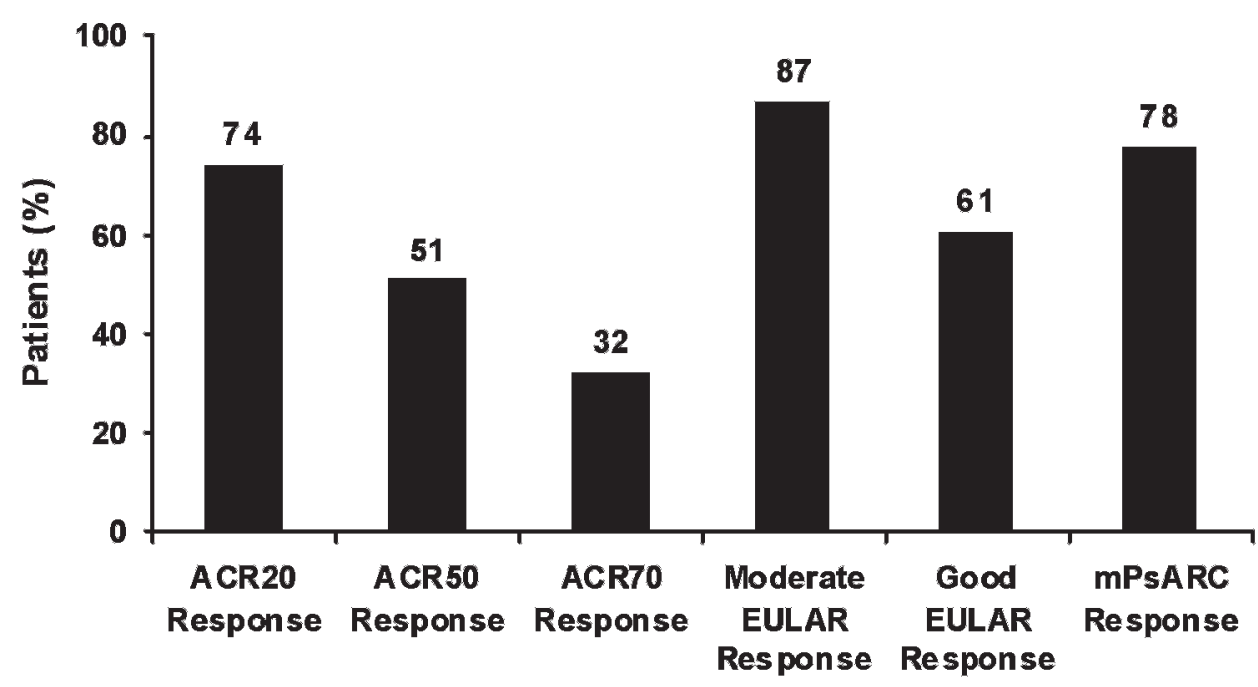

"mild". In the evaluation of individual possible baseline predictors, only a PGA greater than "moderate" (crude OR 2.27, 95\% CI 1.22 to $4.24 ; p=0.011$ ) and a greater PaGA of disease activity (crude OR 1.02, 95\% CI 1.00 to 1.03 ; $p=0.043$ ) were associated with improvement in PGA by $\geqslant 3$ grades.

Predictors of good clinical response for psoriatic nail disorder at week 12 Improvement in NAPSI score by $\geqslant 50 \%$ was experienced by 84 (54.2\%, 9 missing) of 164 patients with a baseline NAPSI score $\geqslant 10$. In the analysis of individual possible predictors, only lower CRP concentration was associated with $\geqslant 50 \%$ improvement in NAPSI score (crude OR 0.79, 95\% CI 0.65 to $0.96 ; p=0.019$ ).

\section{Safety}

There were 21 serious adverse events, including 4 infections, documented for 18 (4.1\%) patients during adalimumab treatment and a 70-day follow-up. Detailed data are summarised in the supplementary material.

\section{DISCUSSION}

The clinical manifestations of PsA in this large study cohort are consistent with the typical pattern of PsA symptoms reported in the literature, and the patient characteristics are representative of those considered eligible for anti-TNF therapy. ${ }^{12} 1314$ After 12 weeks of adalimumab therapy, $74 \%, 51 \%$ and $32 \%$ of the patients experienced ACR20, 50 and 70 responses, respectively. These rates are somewhat greater than those found in randomised controlled trials (RCTs) with adalimumab or other TNF antagonists, which may be because the calculated rates in our study were based on observed values. ${ }^{15-17}$ Moderate and good responses according to EULAR criteria were achieved by $87 \%$ and $61 \%$ of patients, respectively, which is similar to the reported rates in pooled data from two RCTs of other anti-TNF agents (etanercept and infliximab). ${ }^{12}$ The safety profile of adalimumab in this 12 -week study was consistent with results from RCTs of adalimumab for PsA. ${ }^{17}{ }^{18}$

Improvement in psoriasis was clinically relevant, with doubling of the baseline percentage of patients with PGA "clear" or "almost clear" skin at week 12 and a median reduction of the target lesion total plaque score by $67 \%$. The results appear to be within the extent of improvement under anti-TNF therapy when other psoriasis assessment tools were used. ${ }^{15-17}$

This is the first study that has investigated the effect of adalimumab on nail psoriasis. After the relatively short
Figure 2 Percentages of patients achieving Physician Global Assessment of psoriasis (PGA) "clear" or "almost clear" up to week 20. All patients are included, data are from observed cases.

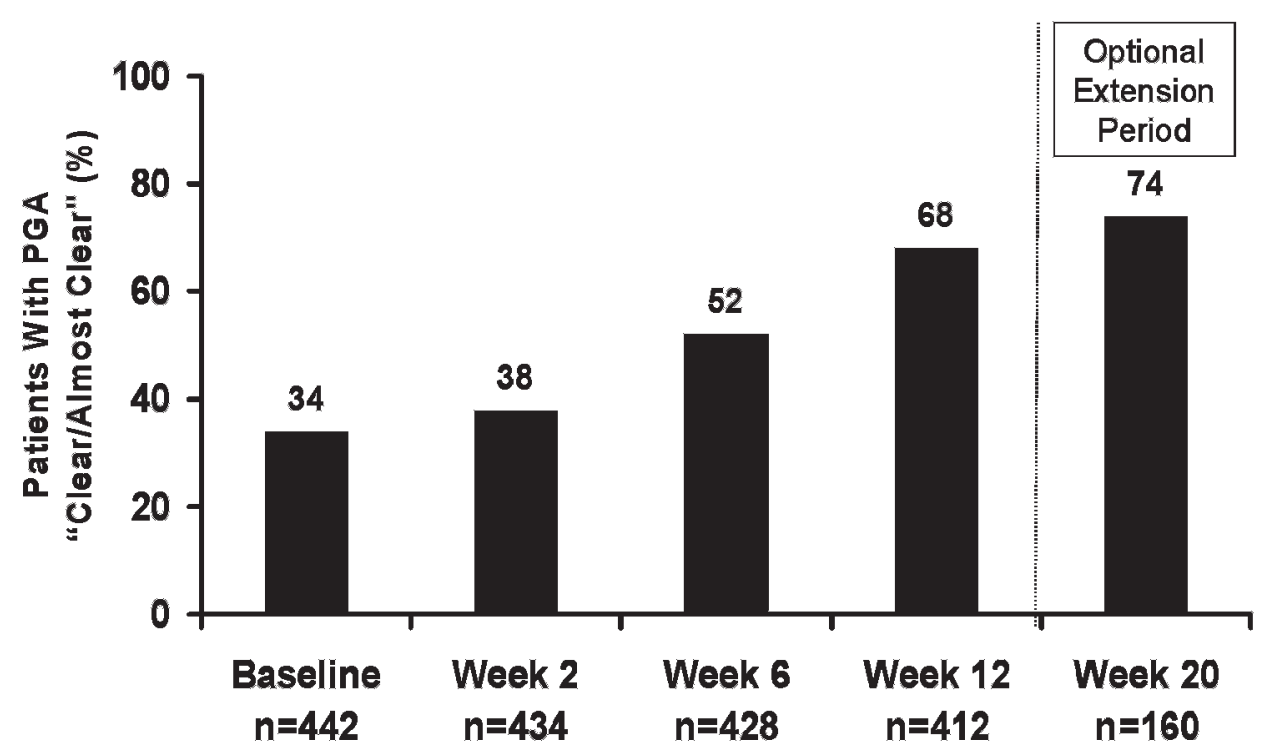


Figure 3 Improvement in median target lesion total plaque score up to week 20 for patients with target lesion $\geqslant 2 \mathrm{~cm}$ in greatest diameter at baseline. Data are from observed cases. ${ }^{*}$ Quartile 1 to quartile 3. ${ }^{* *}$ Median change (means not presented because of skewed distributions).

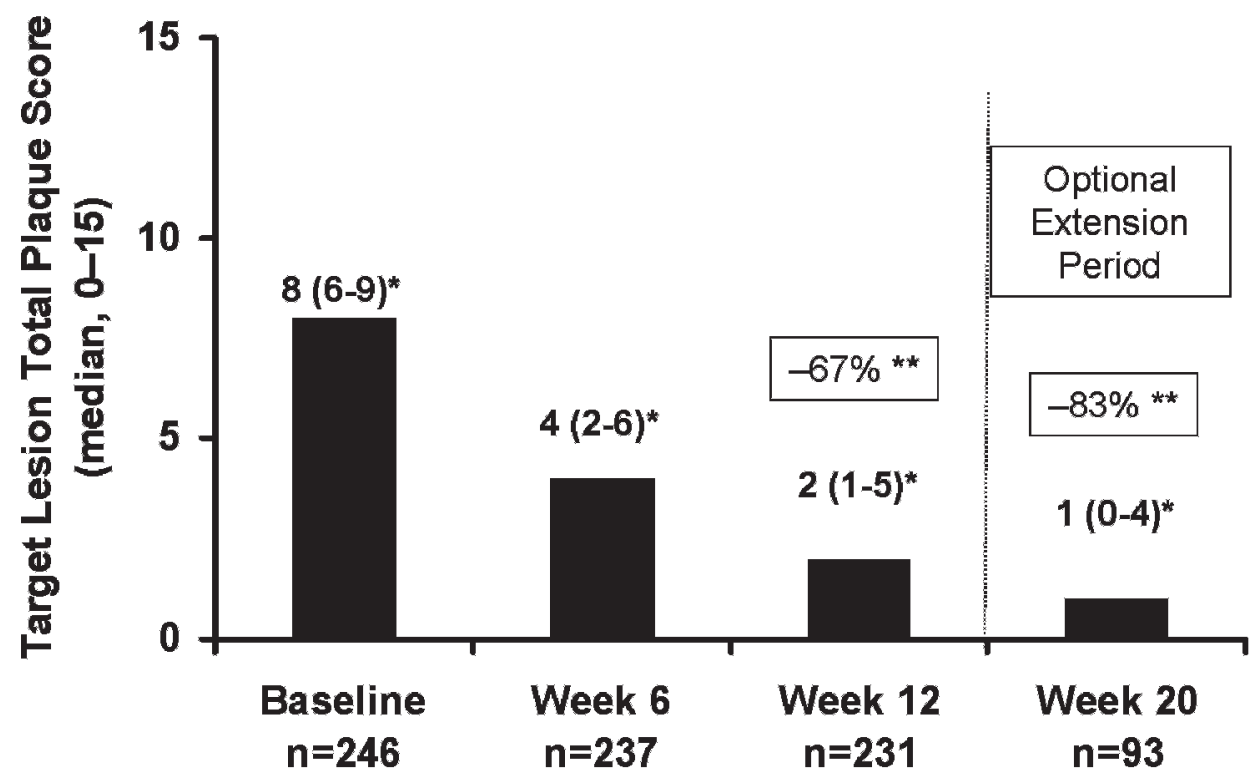

treatment duration of 12 weeks, the median reduction in NAPSI score was $57 \%$. Clearance of psoriasis of the nails was increasing in those patients who continued adalimumab up to week 20. These results are comparable to those reported for infliximab treatment of nail disorder in patients with PsA. ${ }^{19}$

Our results show that good clinical responses in the various manifestations of PsA (ie, joints, skin and nails) are influenced by various predictive factors. Achievement of ACR50 and a good response according to EULAR criteria was more likely when patients had a lower HAQ-DI score and greater pain. Men had a twofold greater chance of achieving ACR50 and good response according to EULAR criteria compared with women. Systemic glucocorticoid therapy, which is generally not recommended for PsA, decreased the chance of a good response. ${ }^{1}$ The chance of achieving ACR50 was also better for patients with greater CRP concentrations and polyarticular PsA, whereas large joint involvement decreased the chance of a good response according to EULAR criteria. These findings are consistent with a predictor analysis in 69 infliximab-treated patients. ${ }^{20}$

Because several of the potential predictors are components of the response criteria, one might expect that greater baseline values would be associated with greater response. With respect to CRP, for example, it is interesting that lower baseline CRP concentrations predict an ACR50 response for patients with rheumatoid arthritis, ${ }^{21}$ which is in contrast to our findings for patients with PsA. For Bath Ankylosing Spondylitis Disease Activity Index 50\% response (BASDAI 50), which does not include CRP, greater baseline CRP concentration predicts a BASDAI 50 response for patients with ankylosing spondylitis. ${ }^{22}$ Thus, CRP appears to be a true biological predictor, and other components of the response criteria may likewise have independent predictive value. Finally, prior use of at least two DMARDs and concomitant treatment with SSZ were associated with the achievement of a good response according to EULAR criteria. Of note, few patients (10.4\%) were concomitantly treated with SSZ (either alone or combined with other DMARDs).

The endpoints of $a \geqslant 3$-grade improvement in PGA and a $\geqslant 50 \%$ improvement in NAPSI score were arbitrarily selected to correspond with ACR50. We found an association between PaGA of disease activity and $\geqslant 3$-grade improvement in PGA. Although greater PGA severity at baseline was associated with
Figure 4 Improvement in median Nail Psoriasis Severity Index (NAPSI) score at weeks 12 and 20 for patients with nail dystrophy (NAPSI $>0$ ) at baseline. Data are from observed cases. ${ }^{*}$ Quartile 1 to quartile 3. ${ }^{* *}$ Median change (means not presented because of skewed distributions).

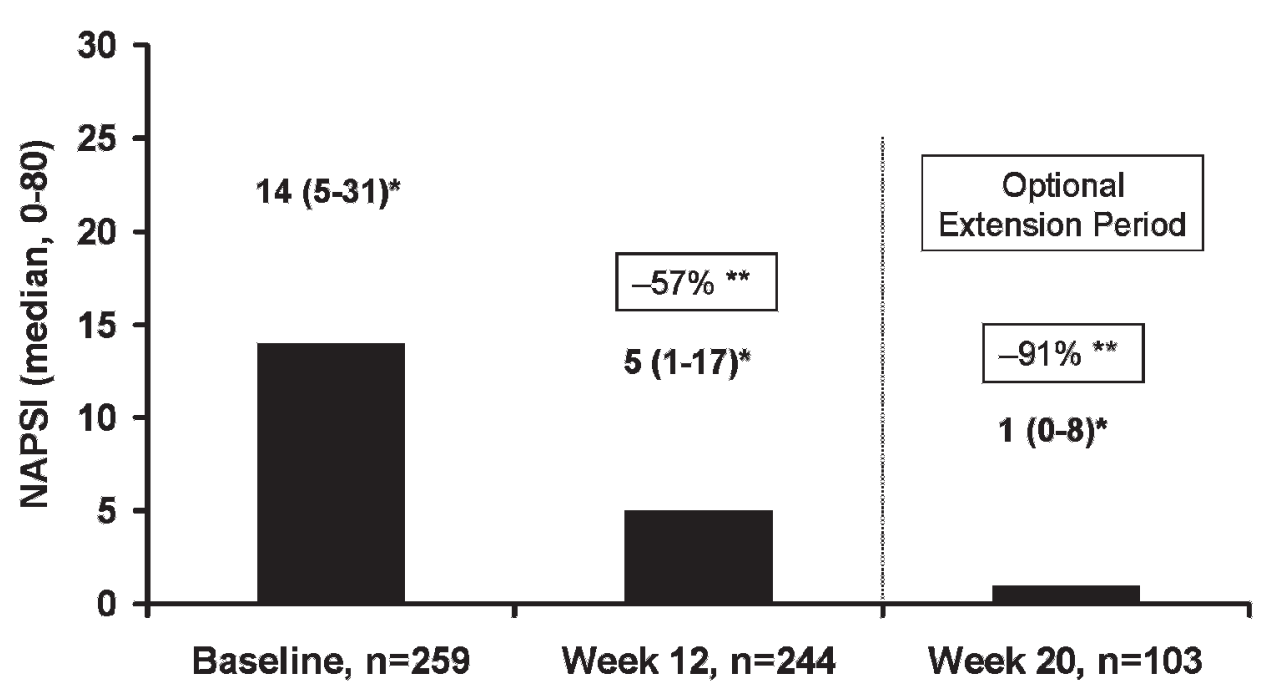


Table 2 Predictors* of good clinical response to adalimumab at week 12, as defined by ACR50 and good response according to EULAR criteria

\begin{tabular}{|c|c|c|c|c|}
\hline \multirow[b]{2}{*}{ Predictors } & \multicolumn{2}{|l|}{ ACR50 response } & \multicolumn{2}{|c|}{ Good response according to EULAR criteria } \\
\hline & OR (95\% Cl) & p Value & OR (95\% Cl) & p Value \\
\hline Sex (male vs female) & 1.77 (1.12 to 2.79$)$ & 0.014 & 2.24 (1.39 to 3.59$)$ & $<0.001$ \\
\hline HAQ-DI (per unit) & $0.39(0.25$ to 0.60$)$ & $<0.001$ & $0.54(0.35$ to 0.85$)$ & 0.007 \\
\hline Systemic glucocorticoids $(\leqslant 10 \mathrm{mg} /$ day $) \dagger$ & $0.57(0.35$ to 0.92$)$ & 0.022 & $0.53(0.32$ to 0.88$)$ & 0.014 \\
\hline Patient's assessment of pain (per 0-100 mm VAS unit) & $1.02(1.01$ to 1.03$)$ & 0.003 & $1.01(1.00$ to 1.03$)$ & 0.027 \\
\hline C-reactive protein (per mg/dl) & $1.15(1.01$ to 1.30$)$ & 0.035 & - & - \\
\hline Swollen joint count $(0-76 ; \geqslant 5$ vs $<5)$ & 2.11 (1.13 to 3.94$)$ & 0.019 & - & - \\
\hline Treatment with SSZ $\dagger$ & - & - & 3.11 (1.35 to 7.12$)$ & 0.007 \\
\hline Prior DMARD use ( $\geqslant 2$ vs $<2)$ & - & - & 1.79 (1.08 to 2.96$)$ & 0.024 \\
\hline
\end{tabular}

*For identification of predictors of ACR50 and good responses according to EULAR criteria, the ensemble of all potential factors was investigated by logistic regression with backward elimination (selection level, 5\%); †yes vs no; \$"large joint" could include the shoulder, elbow, knee, or hip.

ACR50, American College of Rheumatology response criteria, 50\% improvement; DMARD, disease-modifying antirheumatic drug; EULAR, European League Against Rheumatism; HAQ-DI, Health Assessment Questionnaire Disability Index; OR, odds ratio; PsA, psoriatic arthritis; SSZ, sulfasalazine; VAS, visual analogue scale.

greater improvement in PGA, this finding appears to be intrinsically caused by the method and should not be considered a real predictor. $A \geqslant 50 \%$ improvement in NAPSI score was more likely for patients with lower CRP concentrations at baseline, which is opposite to the impact of CRP concentration on achievement of an ACR50 response.

In conclusion, patients with long-term active PsA experienced clinically important improvement in arthritis, psoriasis and psoriatic nail disorder. Low impairment of physical function (lower HAQ-DI score), greater pain, greater CRP concentration, polyarthritis without inflammation of large joints, prior treatment with $>2$ DMARDs, current treatment with SSZ but not glucocorticoids and male sex were factors that increased the chance of achieving substantial clinical improvements.

Acknowledgements: We thank all principal investigators and research nurses who contributed to patient recruitment and data collection in STEREO. We are grateful to the following sites that have enrolled at least five patients with PsA. Belgium: Dr V Smith, Dr F Van den Bosch (Gent); Denmark: Dr P Holck (Silkeborg); Dr P Mosborg Petersen (Randers); Professor Dr 0 R Madsen (Hellerup); Finland: Dr P Jaevinen (Hyvinkää); Dr T Yli -Kerttula (Turku); Dr L Paimela (Helsinki); France: Professor Dr B Combe (Montpellier); Professor Dr L Euller-Ziegler (Nice); Professor Dr P Fardelle (Amiens); Professor Dr RM Flipo (Lille); Professor P Goupille (Tours); Professor Dr Y Maugars (Nantes): Germany: Professor Dr G Burmester (Berlin): Professor Dr E Gromnica-Ihle (Berlin); Professor Dr G Hein (Jena); Dr P Hrdlicka (Chemnitz); Professor Dr Kaltwasser (Frankfurt); Professor Dr K Krüger (München); Dr R Kurthen (Aachen); Professor Dr B Manger (Erlangen); Dr L Meier (Hofheim am Taunus); Professor Dr U Müller-Ladner (Bad Nauheim); Professor Dr HH Peter (Freiburg); Dr R Spreckeler (Zeven); Professor Dr HP Tony (Würzburg); Dr U von Hinüber (Hildesheim); Dr S Wassenberg (Ratingen); Professor Dr J Wollenhaupt (Hamburg); Ireland: Professor Dr 0 FitzGerald (Dublin); Norway: Dr K Helgetveit (Oslo); Dr K Mikkelsen (Lillehammer); Dr E Rødevand (Trondheim); Dr DM Soldal (Arendal); Sweden: Dr H Bolinder (Stockholm); Dr G Holmstroem (Oskarström): Dr A Jalal (Öerebro): Professor Dr Ronald van Vollenhoven (Stockholm); UK: Dr RG Cooper (Manchester); Dr B Kirkham (London); Dr N McHugh (Bath); Dr R Reece (Lindley Huddersfield); Dr D O'Reilly (Suffolk). We also thank Dr Christine Frank for study management (Abbott, Germany); Christa Zaiti-Runkel for data management; and Angelika Freitag and Anja Bruhn for statistical programming (both Abbott, Germany). We also thank Dana L Randall (Arbor Communications, Ann Arbor, Michigan, USA) and Michael A Nissen, ELS, of Abbott Laboratories, for editing and publication management support in the development and revision of this manuscript.

Funding: The editing and publication management support in the development and revision of this manuscript was funded by Abbott Laboratories.

Competing interests: FvdB has received speaker's bureau honorarium and/or has served as a consultant for Abbott Laboratories, Schering-Plough, UCB and Wyeth. OF has given lectures at the request of Abbott and Wyeth, and has also received grant support from both companies. PH is a government employee. RvV has received research grants and consulting fees from Abbott, Bristol-Myers-Squibb, Centocor, Roche, Wyeth and Schering-Plough. ML-R has given lectures/organised education events at the request of Abbott, Roche, Wyeth, Schering-Plough and Bristol-Myers-Squibb, and has received research grants from Schering-Plough as well as consulting fees from Centocor, BristolMyers-Squibb, Roche and Novartis. ML-R has also served as advisory board members for Abbott, Schering-Plough, Roche and Bristol-Myers-Squibb. BM has received honoraria as speaker or consultant from the following companies: Abbott, Bristol-MyersSquibb, Essex, Genzyme, Novartis, 4SC, Roche, UCB Pharma and Wyeth. PG has received research grants from Abbott and Wyeth; consulting fees from Abbott, Wyeth, Schering-Plough and Roche, and has been an advisory board member for Abbott, Wyeth, Schering-Plough, Roche and Bristol-Myers-Squibb. NM has received an unrestricted research grant from Abbott for a study of long-term outcomes in patients with psoriatic arthritis. ER has nothing to disclose. MK, MF and HK are full time employees of Abbott, an affiliate of Abbott Laboratories and hold shares of Abbott stock. SK is a contractor of Abbott GmbH.

Trial registration number: NCT00235885

The STEREO Study Group included experts from academic institutions in Europe and members of Abbott Laboratories who designed the clinical trial. Clinical data were collected and analysed by Abbott Laboratories. All authors contributed to manuscript development and reviewed and approved the content of the submitted manuscript.

Provenance and peer review: Not commissioned; externally peer reviewed.

\section{REFERENCES}

1. Ritchlin CT, Kavanaugh A, Gladman DD, et al. Treatment recommendations for psoriatic arthritis. Ann Rheum Dis 2009;68:1387-94.

2. Furst DE, Breedveld FC, Kalden JR, et al. Updated consensus statement on biological agents for the treatment of rheumatic diseases, 2007. Ann Rheum Dis 2007:66/Suppl 3):iii2-22.

3. Felson DT, Anderson JJ, Boers M, et al. American College of Rheumatology. Preliminary definition of improvement in rheumatoid arthritis. Arthritis Rheum 1995; 38:727-35.

4. Prevoo ML, van't Hof MA, Kuper HH, et al. Modified disease activity scores that include twenty-eight-joint counts. Development and validation in a prospective longitudinal study of patients with rheumatoid arthritis. Arthritis Rheum 1995:38:44-8.

5. van Gestel AM, Prevoo ML, van't Hof MA, et al. Development and validation of the European League Against Rheumatism response criteria for rheumatoid arthritis. Comparison with the preliminary American College of Rheumatology and the World Health Organization/International League Against Rheumatism criteria. Arthritis Rheum 1996;39:34-40.

6. Clegg DO, Reda DJ, Mejias E, et al.Comparison of sulfasalazine and placebo in the treatment of psoriatic arthritis. A Department of Veterans Affairs Cooperative Study. Arthritis Rheum 1996:39:2013-20.

7. Blackmore MG, Gladman DD, Husted J, et al. Measuring health status in psoriatic arthritis: the Health Assessment Questionnaire and its modification. J Rheumatol 1995:22:886-93.

8. Gladman DD, Helliwell P, Mease PJ, et al. Assessment of patients with psoriatic arthritis: a review of currently available measures. Arthritis Rheum 2004:50:24-35.

9. Rich P, Scher RK. Nail Psoriasis Severity Index: a useful tool for evaluation of nail psoriasis. J Am Acad Dermatol 2003:49:206-12.

10. Finlay AY, Khan GK. Dermatology Life Quality Index (DLOI) - a simple practical measure for routine clinical use. Clin Exp Dermatol 1994;19:210-6.

11. Mease PJ, Antoni CE, Gladman DD, et al. Psoriatic arthritis assessment tools in clinical trials. Ann Rheum Dis 2005;64(Suppl 2):ii49-54.

12. Fransen J, Antoni C, Mease PJ, et al. Performance of response criteria for assessing peripheral arthritis in patients with psoriatic arthritis: analysis of data from randomised controlled trials of two tumour necrosis factor inhibitors. Ann Rheum Dis 2006:65:1373-8.

13. Gladman DD, Antoni C, Mease P, et al. Psoriatic arthritis: epidemiology, clinical features, course, and outcome. Ann Rheum Dis 2005:64(Suppl 2):ii14-7.

14. Taylor WJ, Marchesoni A, Arreghini M, et al. A comparison of the performance characteristics of classification criteria for the diagnosis of psoriatic arthritis. Semin Arthritis Rheum 2004:34:575-84. 
15. Mease PJ, Goffe BS, Metz J, et al. Etanercept in the treatment of psoriatic arthritis who had failed disease modifying antirheumatic drug therapy. Lancet 2000;356:385-90.

16. Antoni CE, Kavanaugh A, Kirkham B, et al. Sustained benefits of infliximab therapy for dermatologic and articular manifestations of psoriatic arthritis: results from the infliximab multinational psoriatic arthritis controlled trial (IMPACT). Arthritis Rheum 2005;52:1227-36.

17. Mease PJ, Gladman DD, Ritchlin CT, et al. Adalimumab for the treatment of patients with moderately to severely active psoriatic arthritis. Arthritis Rheum 2005; 52:3279-89.

18. Gladman D, Mease P, Ritchlin CT, et al. Adalimumab for long-term treatment of psoriatic arthritis. Arthritis Rheum 2007;56:476-88.
19. Rigopoulos D, Gregoriou S, Stratigos A, et al. Evaluation of the efficacy and safety of infliximab on psoriatic nails: an unblinded, nonrandomized, open-label study. Br J Dermatol 2008;159:453-6.

20. Gratacós J, Casado E, Real J, et al. Prediction of major clinical response (ACR50) to infliximab in psoriatic arthritis refractory to methotrexate. Ann Rheum Dis 2007;66:493-7.

21. Burmester GR, Mariette X, Montecucco C, et al. Adalimumab alone and in combination of disease modifying anti-rheumatic drugs for the treatment of rheumatoid arthritis in clinical practice: the Research in Active Rheumatoid Arthritis (ReAct) trial. Ann Rheum Dis 2007;66:232-9.

22. Rudwaleit M, Claudepierre P, Wordsworth $P$, et al. Effectiveness, safety, and predictors of good clinical response in 1250 patients treated with adalimumab for active ankylosing spondylitis. J Rheumatol 2009;36:801-8. 